\title{
Análisis de determinantes de las prácticas educativas parentales en hogares con hijos entre primero y cuarto año de educación básica de la ciudad de Guayaquil
}

\section{Analysis of determinants of parental educational practices in homes with children between first and fourth year of basic education of the city of Guayaquil}

Jorge Izaguirre Olmedo

Universidad Internacional del Ecuador, Ecuador

Viviana Medina Vergara

Universidad Internacional del Ecuador, Ecuador

Autor para correspondencia: jorgeandres.izaguirre@gmail.com, vimedinave@uide.edu.ec Fecha de recepción: 24 de Mayo de 2017 - Fecha de aceptación: 10 de Agosto de 2017

\section{Resumen}

Este trabajo consiste en la estimación de un modelo de regresión lineal general que busca evaluar la significancia de variables asociadas a las Prácticas Educativas Parentales (PEP), así como el efecto generado en pendiente y magnitud. Para su realización se han utilizado datos obtenidos a través de la aplicación de una encuesta a padres de familia y apoderados de niños en escuelas privadas y fiscales que cursan entre el primero y cuarto año de educación básica. Entre los resultados obtenidos se desea observar si existe diferencia estadística entre las PEP en hogares con diferente posición socioeconómica y cultural.

Palabras Clave: prácticas educativas parentales; regresión; determinantes; significancia

\begin{abstract}
This paper refers to an estimation of a general lineal regression model that tends to evaluate the significance of variables associated to Educational Practices of Parents, as well as the effect generated in trend and magnitude. For developing this work, it is been used a data provided for a survey to parents or responsible of children in private and public schools between the first and fourth grade of basic education. In the results, it is expected to determine if there is a statistic difference between Educational Practices of Parents and economic and cultural level of the family.
\end{abstract}

Key words: educational practices of parents; regression; factors; significance 


\section{Introducción}

Las Prácticas Educativas Parentales (PEP), corresponden a una de las dimensiones de las Estrategias Educativas Familiares (EEF), las cuales han sido definidas por Bourdieu \& Wacquant (1993/1995) como "el despliegue activo de líneas de acción objetivamente orientadas que obedecen a regularidades y conforman patrones coherentes y socialmente inteligibles, aun cuando no siguen reglas conscientes o apuntan a las metas premeditadas determinadas por un estratega". Gubbins \& Ibarra (2016) explican esta definición como el conjunto de estrategias que definen las familias por cuenta propia, a través de sus creencias y aspiraciones, para apoyar a la educación de sus hijos.

El estudio de las EEF permiten determinar el grado de incidencia que existe entre las actividades de las familias y las diferencias cognitivas o de educación que puedan alcanzar los niños en sus respectivas escuelas. En este sentido, las EEF incluyen cuatro dimensiones que son: el capital socio-cultural, la Disposición Parental hacia la Escuela, las Aspiraciones Parentales y las Prácticas Educativas Parentales (Gubbins \& Ibarra, 2016).

La importancia del Involucramiento Familiar en la educación del niño fue puesta en relieve por Epstein (2013) al demostrar que siendo el estudiante el eje central, es corresponsabilidad de escuela, familia y comunidad el establecimiento de estrategias que ayuden al desarrollo cognitivo del niño. Este involucramiento familiar es uno de los factores que tienen mayor influencia en el logro académico (Weiss, Bouffard, Bridgall, \& Edmund, 2009). Por su parte, López \& Caspe (2014) definieron al involucramiento familiar como la corresponsabilidad de familia y escuela en la educación del niño, la cual debe mantenerse hasta la adultez temprana.

El Ministerio de Educación del Ecuador (2015) señala la importancia de las relaciones de las familias con las escuelas en contextos de aprendizaje. Anteriormente el involucramiento de las familias se limitaba a organizaciones en las escuelas con fines específicos, no obstante con los avances en educación inicial, la responsabilidad familiar ha estado tomando mayor relevancia debido a los resultados favorables del involucramiento sobre la educación (Robledo \& García, 2009).

Las investigaciones sobre EEF han estado orientadas a estudios descriptivos en las cuatro dimensiones anteriormente mencionadas. Como hipótesis previa se podría pensar que estas cuatro dimensiones se encuentran correlacionadas entre sí. De esta manera, el objetivo central de esta investigación es medir el efecto que tienen el capital socio-cultural de los padres, la disposición parental y las aspiraciones parentales; sobre las PEP, las cuales, acorde a los resultados de involucramiento familiar, tendrán un resultado positivo sobre la educación del niño.

\section{Metodología}

Esta investigación tiene un enfoque cuantitativo - causal. Para la obtención de la data se levantaron encuestas a padres de familia y apoderados de niños que estudian entre el primero y cuarto año de educación básica en la ciudad de Guayaquil, durante los meses de diciembre del 2016 y enero del 2017. Se estratificó la muestra para obtener información de escuelas públicas y privadas, de tal forma que puedan alcanzarse distintos niveles socioeconómicos. 
Para la ejecución del cuestionario se procedió a solicitar autorización a los directores de las escuelas y se encuestaron a los padres o apoderados durante las reuniones para entrega de libretas. El cuestionario fue auto-administrado para eliminar el sesgo de información debido a las preguntas sensibles referentes a ingresos y gastos. Las preguntas, en total 31 con varios literales, se podrían clasificar en cinco grupos: caracterización del estudiante, el apoderado y la familia, capital socio-cultural de la familia, disposición parental, aspiraciones parentales y prácticas educativas.

A excepción de las preguntas referentes a la caracterización del estudiante, apoderado y familia, el cuestionario fue elaborado en base a afirmaciones que se respondían en una escala de Likert de 4 niveles que incluían: nunca, casi nunca, ocasionalmente, muy frecuentemente. Posteriormente, se agruparon las respuestas y se crearon índices que serán explicados en la sección de resultados.

Para el análisis de los datos se utilizó el paquete SPSS y como método de estimación se realizó una regresión lineal general utilizando como variable dependiente el logaritmo del índice de las prácticas educativas familiares y se evaluaron las siguientes variables independientes: edad del apoderado, ingreso, nivel de educación, frecuencia de lectura, índice de capital socio-cultural, índice de disposición parental e índice de aspiraciones parentales.

\section{Revisión de la Literatura}

Acorde a Bourdieu \& Wacquant (1993/1995), citados por Gubbins \& Ibarra (2016), las EEF son un "despliegue activo de líneas de acción objetivamente orientadas que obedecen a regularidades y conforman patrones coherentes y socialmente inteligibles, aun cuando no siguen reglas conscientes o apuntan a las metas premeditadas determinadas por un estratega" (Pág. 1). Esta definición refuerza el concepto propuesto por Bourdieu \& Passeron (1977), quienes indicaron que las EEF son representaciones mentales y prácticas de la familia que nacen del contexto. Gubbins \& Ibarra (2016) dividen las EEF en cuatro dimensiones: i) capital económicocultural de los padres; ii) disposición parental hacia la escuela; iii) aspiraciones parentales; y iv) prácticas educativas parentales.

El capital económico - cultural de una familia puede generar diferencias en el desarrollo cognitivo del niño y las estrategias de los padres para su educación (Weiss, Bouffard, Bridgall, \& Edmund, 2009). El capital económico se refiere al nivel de ingreso de la familia, mientras que el capital cultural recae en el nivel de educación de los padres o la frecuencia de actividades culturales de la familia. Mientras más alto es el nivel cultural de los padres, es mayor el número de interacciones positivas que tiene la familia (Gubbins \& Ibarra, 2016).

Bordieu (1977) indicaba que las familias con bajos recursos tienden a no poseer el capital cultural de aquellas familias de clase social alta. Weiss et al. (2009) por su parte indica que dichas familias con bajo capital económico son más propensas a no participar de actividades organizadas por las escuelas y desconocer las políticas institucionales. Esta idea refuerza lo que se había indicado en investigaciones anteriores referentes a que la pobreza impedía o limitaba las prácticas de involucramiento familiar (Mc Loyd, V., 1990; Mc Loyd, V., Jayaratne, T., Ceballo, 
R., y Borquez, J., 1994; citados por Weiss et. al., 2009). Esta falta de involucramiento podría estar relacionada a una auto-discriminación, así como problemas más puntuales como falta de flexibilidad laboral, problemas de movilidad, entre otros.

Lareau (2003), citado por Weiss et. al (2009), indica que las diferencias en las clases sociales de los padres determinan brechas en el capital cultural de los hijos. Sobre esto, Weiss et. al (2009) indican que las actividades extracurriculares no son percibidas como aportes positivos para los hijos por parte de los padres de clase social baja, mientras que el resultado es contrario en clases sociales medias y altas. Por su parte, Gubbins \& Ibarra (2016) concluyen que los padres con menor capital económico y cultural, tienden a tener menores cantidades de actividades formales y no formales con la escuela. Esto supone la hipótesis previa de que debería existir una correlación entre el ingreso familiar y las prácticas educativas familiares.

En lo que corresponde al componente de disposición parental hacia la escuela, podría definirse como el grado de inclinación de los padres de familia hacia la educación formal y su capacidad de respuesta activa a los requerimientos de la escuela. Esta inclinación denota el nivel de expectativas y aceptación de los padres con la educación recibida por los hijos. Entre los ejemplos de dicha disposición se puede mencionar: la asistencia a reuniones, la comunicación constante con los profesores, la participación en actividades extracurriculares, la ayuda a los hijos ante cualquier problema, entre otros (Bourdieu \& Wacquant, 1993/1995; citado por Gubbins \& Ibarra, 2016).

Epstein (1995), citado por Rivera \& Milicic (2006), señala que los hogares que cuentan con padres que se preocupan por la educación de los hijos y los alientan a mejorar en esta parte, consiguen que dichos hijos sobresalgan en la parte académica y muestren una actitud positiva hacia la escuela. Bajo esta idea, Sallés \& Ger (2011) señalan que la familia y la comunidad son responsables de involucrarse por el desarrollo de competencias parentales. Sin embargo, en la práctica, muchos padres no optan por asistir a reuniones debido a que los docentes las convocan sólo con carácter informativo, para solicitar aportes económicos o material didáctico y para dar quejas del comportamiento de los estudiantes (Gubbins, 2012).

En referencia al tercer componente, las aspiraciones parentales, el Programa de las Naciones Unidas para el Desarrollo (2000), citado por Rivera \& Milicic (2006), define a dichas aspiraciones como "las representaciones que se hacen los individuos y los grupos acerca del estado de las cosas, personales o sociales, que desean para el futuro y que caracterizan como lo mejor" (pág. 3). En esta investigación se sigue la línea de pensamiento propuesta por Gubbins \& Ibarra (2016), quienes enfocan las aspiraciones en el ámbito de la familia - escuela. En este sentido, las aspiraciones serían las expectativas de los padres sobre la formación educativa de sus hijos y las competencias que pueda proveer la escuela.

Jeynes W. (2003), citado por Weiss et al. (2009), evidenció que existe una relación positiva del nivel de expectativas que tienen los padres sobre la educación de los hijos y el logro académico alcanzado por ellos. Así mismo, Catsambis (2001), citado por Weiss et. al (2009), indica que la interacción de padres e hijos sobre la importancia de la educación presente y futura arroja resultados escolares positivos. 
Finalmente, en cuanto al componente de las prácticas parentales, Darling \& Steinberg (1993), citado por Rivera \& Milicic (2006), las definen como conductas concretas que los padres desempeñan en su rol y que van direccionadas a una meta. Según Baumrind (1971) se identifican tres estilos de prácticas parentales: permisivos, autoritativos y autoritarios. Entre estos tres estilos, el autoritativo es el que mejores resultados mostraba, perdurando hasta la adolescencia (Weiss, Bouffard, Bridgall, \& Edmund, 2009).

Gubbins \& Ibarra (2016) en su investigación desarrollada en Chile, demostraron con estadísticas descriptivas que las EEF difieren acorde a niveles socioeconómicos y culturales, sin profundizar la investigación en dimensiones o relaciones entre los componentes de las EEF. Este sería el punto de partida de la presente investigación, la cual pretende medir el grado de incidencia del capital económico - cultural, la disposición parental hacia la escuela y las aspiraciones parentales, sobre las prácticas educativas familiares.

\section{Resultados}

Para un correcto análisis de los resultados, en esta sección se iniciará con un desglose de las variables, así como las transformaciones que se le dieron a cada una para la realización de los cálculos.

\section{Variables Independientes}

El primer grupo de variables independientes corresponden al capital económico - cultural de los padres. Las variables que se utilizaron fueron la edad, el ingreso promedio del hogar, el nivel de integración del hogar y la frecuencia de lectura del apoderado.

Tabla 1: Estadísticos descriptivos de la Edad del encuestado

\begin{tabular}{lrrrrr}
\hline & N & Mínimo & Máximo & Media & Desv. típ. \\
\hline Edad del encuestado & 400 & 21 & 70 & 34,85 & 7,411 \\
N válido (según lista) & 400 & & & & \\
\hline
\end{tabular}

Como puede observarse en la tabla 1, la edad mínima de los apoderados encuestados fue de 21, la edad máxima llegó a los 70 años y el promedio se ubicó en 34,85. La distribución mostró una desviación típica con respecto a la medio de 7,4.

Tabla 2: Ingreso Promedio Mensual del Hogar

\begin{tabular}{llrrrr}
\hline & & & & \\
& & Frecuencia & Porcentaje & Porcentaje válido & Porcentaje acumulado \\
\hline Válidos & Menos de $\$ 470$ & 135 & 33,8 & 33,8 & 33,8 \\
& De $\$ 471$ a $\$ 1,000$ & 53 & 13,3 & 13,3 & 47,1 \\
& De $\$ 1,001$ a $\$ 2,000$ & 90 & 22,5 & 22,6 & 69,7 \\
& Más de $\$ 2,000$ & 121 & 30,3 & 30,3 & 100,0 \\
\hline
\end{tabular}




\begin{tabular}{llrrr}
\hline & Total & 399 & 99,8 & 100,0 \\
Perdidos & Sistema & 1 &, 3 & \\
Total & & 400 & 100,0 & \\
\hline
\end{tabular}

La tabla 2 muestra el desglose de la variable de ingreso. Esta variable se recogía por rangos dentro de la encuesta, por lo que no es posible manejarla en niveles cuantitativos. Se observa que el 33,8\% de la muestra indica tener ingresos inferiores a los $\$ 470$; el 13,3\% de la muestra se ubica entre $\$ 471$ y $\$ 1,000$; el $22,5 \%$ reporta tener ingresos entre $\$ 1,001$ y $\$ 2,000$; y el $30,3 \%$ indica tener ingresos superiores a los $\$ 2,000$.

Para analizar el nivel de integración del hogar, se realizaron varias preguntas referentes a las actividades que realizan las familias. En este sentido se consultó sobre: la asistencia a conciertos, a juegos o espectáculos deportivos, a eventos culturales y paseos turísticos en familia. Las respuestas se dieron en cuatro niveles a través de escalas de Likert y se procedió con el cálculo de promedios para encontrar un índice referente al nivel de integración del hogar. Se espera que las familias que muestren mayor nivel de integración, tengan una relación positiva con la cantidad o nivel de prácticas educativas que realicen. La ilustración 1 muestra el histograma del nivel de integración del hogar.

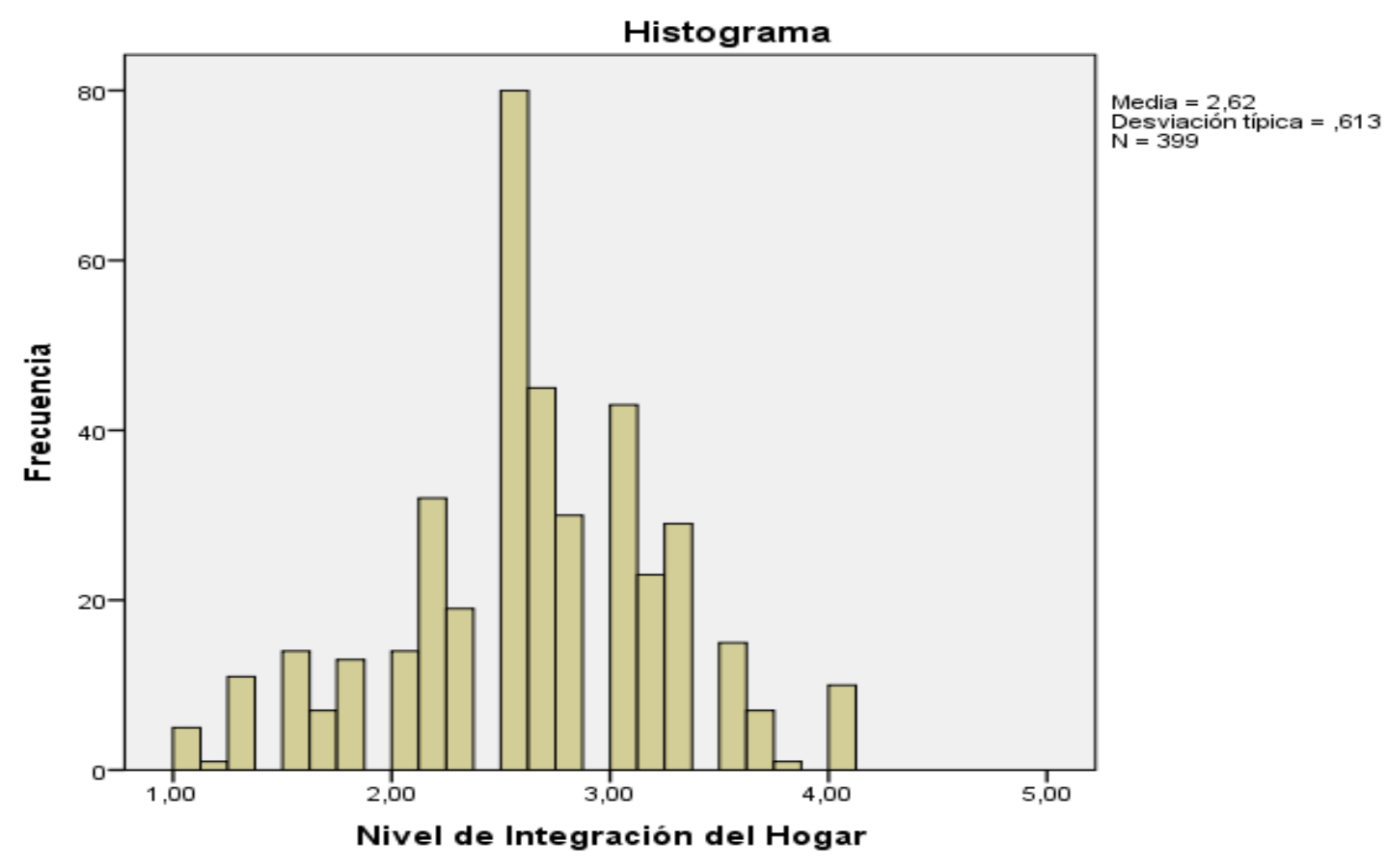

Ilustración 1: Nivel de Integración del Hogar

En cuanto a la frecuencia de lectura del apoderado, la tabla 3 muestra los resultados. El $18,3 \%$ reporta leer todos los días. El 29,3\% reporta leer $1 \mathrm{vez}$ a la semana al menos. El 11,3\% reporta leer cada 15 días. El 7,3\% reporta leer una vez al mes. El porcentaje restante lee en menor cantidad o no lee. 


\section{Tabla 3: Frecuencia de Lectura}

\begin{tabular}{llrrrr}
\hline \multicolumn{5}{c}{ ¿Con qué frecuencia acostumbra usted a leer libros? } \\
& Frecuencia & Porcentaje & Porcentaje válido & Porcentaje acumulado \\
\hline Válidos & Todos los días & 73 & 18,3 & 18,3 & 18,3 \\
& 117 & 29,3 & 29,3 & 47,5 \\
& 1 vez por semana & 45 & 11,3 & 11,3 & 58,8 \\
Cada 15 días & 29 & 7,3 & 7,3 & 66,0 \\
1 vez al mes & 30 & 7,5 & 7,5 & 73,5 \\
Cada 2 o 3 meses & 23 & 5,8 & 5,8 & 79,3 \\
Cada 4 o 5 meses & 28 & 7,0 & 7,0 & 86,3 \\
Cada 6 meses o más & 55 & 13,8 & 13,8 & 100,0 \\
Nunca (no lee libros) & 400 & 100,0 & 100,0 & \\
Total & & & &
\end{tabular}

El segundo índice o componente de variables independientes corresponde a la disposición parental hacia la escuela. En este índice su consultó a los entrevistados sobre: frecuencia de asistencia a las reuniones de apoderados convocados por el establecimiento educacional, asistencia a entrevista con dirigente de grado para analizar conducta o rendimiento académico del estudiante, ayuda al profesor en la sala de clases, voluntariado para mejorar la infraestructura del establecimiento educacional, voluntariado en actividades organizadas y realizadas dentro del establecimiento educacional, acompañamiento al profesor en actividades sociales o culturales del curso realizadas fuera del establecimiento educacional, voluntariado en la biblioteca o en el centro de recursos del aprendizaje del establecimiento educacional, asistencia a actividades extraprogramáticas organizadas por los apoderados, asistencia a actos y celebraciones organizadas por el establecimiento educacional, conformación del comité de grado, entre otras. Se pidió evaluar su participación en una escala del uno al cuatro, siendo uno el menor nivel de disposición parental en cada categoría y cuatro el mayor nivel. Se realizó un promedio para encontrar el índice de disposición parental. Los resultados se muestran en la ilustración 2. 


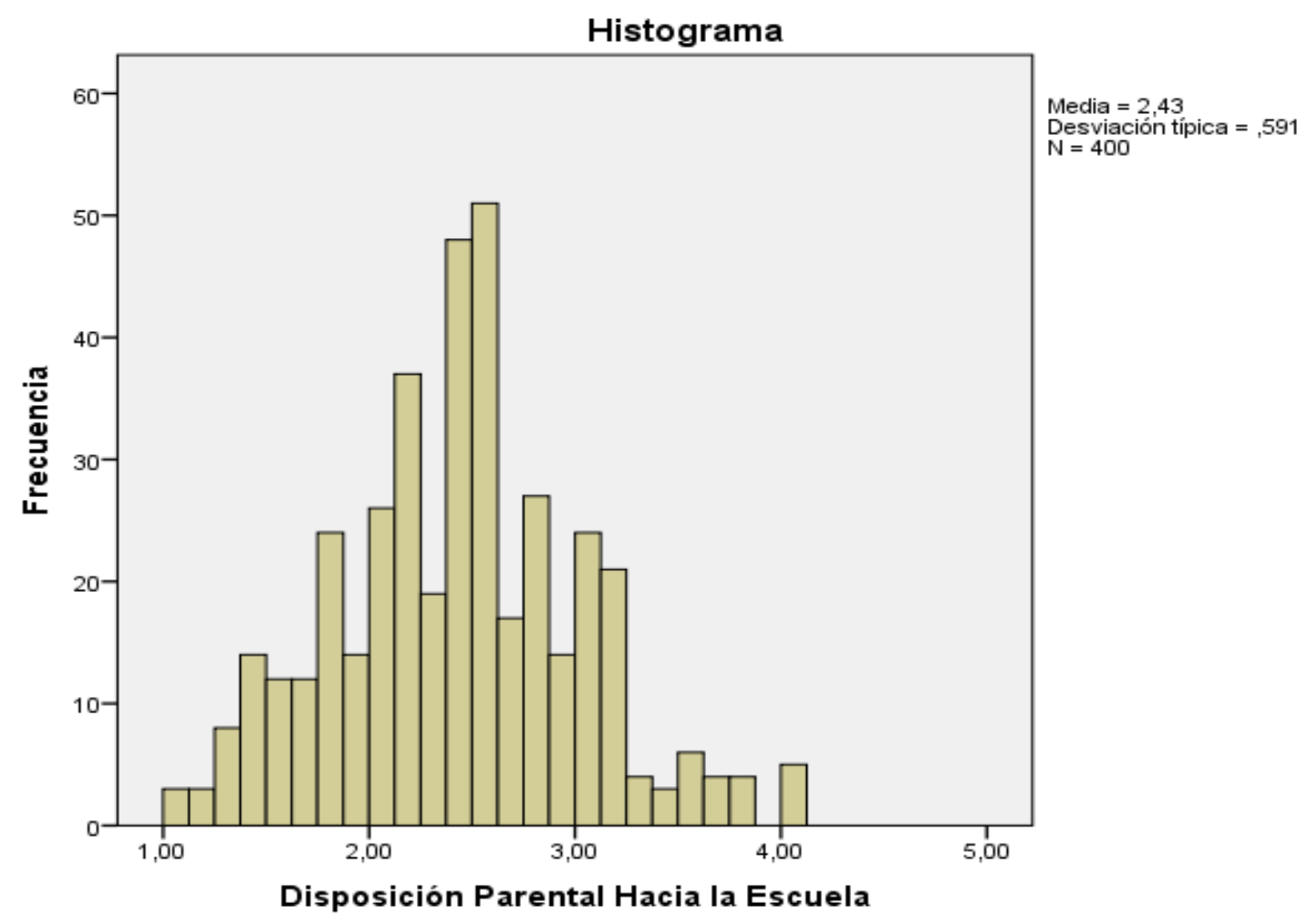

Ilustración 2: Disposición Parental hacia la Escuela

Otro índice utilizado como variable independiente corresponde a las aspiraciones parentales. Para la construcción de este índice se consideraron las siguientes expectativas de los padres o apoderados con respecto a la escuela: que preparen al estudiante para que rinda una buena prueba ENES y logre ingresar a la universidad; que desarrollen su capacidad de inteligencia, crítica y reflexión; que lo formen como una persona autónoma y responsable; y, que le entreguen los conocimientos necesarios para que alcance un buen trabajo. Los resultados del índice se muestran en el siguiente histograma. 


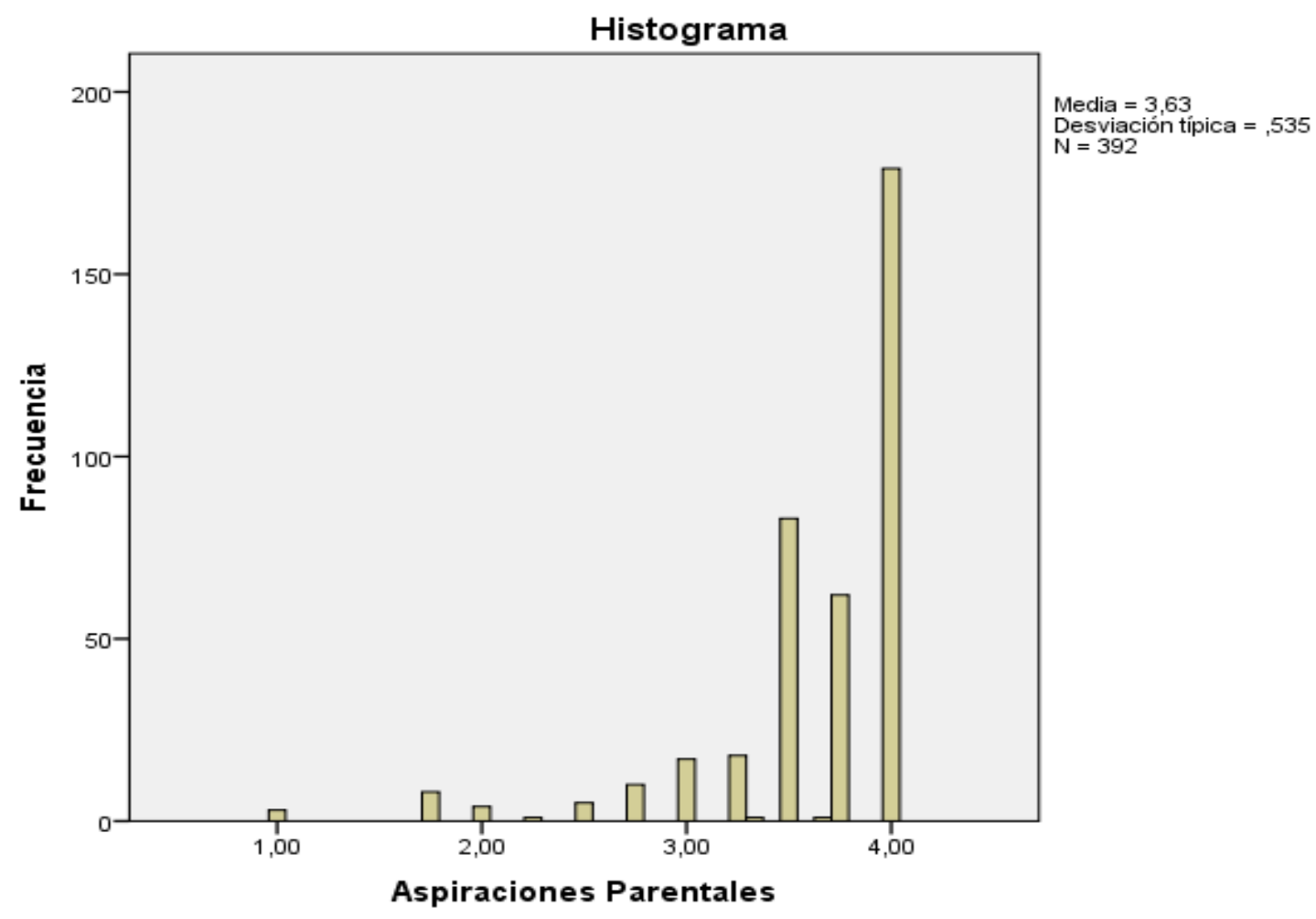

Ilustración 3: Aspiraciones Parentales

Como puede observarse en el histograma, existe una clara tendencia de los padres o apoderados a tener altas expectativas de la educación de la escuela con los hijos. Se esperaría que esta variable muestre una correlación positiva con las prácticas educativas.

Finalmente, se utilizó una variable denominada Nivel de Exigencia, la cual es un índice que refleja la cantidad de controles que realizan los padres en la educación de los hijos. Estos controles no se consideraron prácticas educativas e incluyen: control de horarios para tareas, lugares específicos para desarrollar deberes, definición de horarios para ver televisión, entre otros.

\section{Variable Dependiente}

La variable dependiente utilizada para esta investigación es un índice de las prácticas educativas familiares que ha sido trabajado en logaritmos para una mejor interpretación de los resultados. De esta manera, se podrá concluir sobre incrementos o reducciones porcentuales del índice de prácticas educativas utilizadas por los padres.

Las prácticas educativas de los padres para con los hijos, a las que se hacen referencia en este trabajo son:

- $\quad$ Leerle libros. 
- Incentivarle la lectura por cuenta propia.

- Contarle cuentos inventados.

- Discutir con él/ella sobre temas de actualidad.

- Dedicar un tiempo para conversar con su hijo.

- Hablar con él/ella sobre lo que está leyendo por cuenta propia.

- Conversar con él/ella sobre la importancia de la escuela.

- Incentivarle que escriba pequeños textos (cuentos, poemas, historias).

- Enseñarle a organizar su día con tiempos y lugares para estudiar.

- Revisarle las tareas escolares.

- Estudiar con él/ella para los controles y pruebas.

- Enseñarle hábitos y estrategias de estudio.

- Analizar en conjunto los logros y dificultades académicas.

- Contactarlo con otros estudiantes u otros adultos que sepan más y lo apoyen en sus tareas o estudios.

- Plantearle operaciones matemáticas (medir cosas, contar, restar).

- Incentivarle que pida ayuda a compañeros con buen rendimiento escolar.

- Incentivarlo a que resuelva de manera autónoma sus actividades escolares.

- Resolver sus dudas mientras realiza sus actividades escolares.

- Sentarse personalmente a trabajar con el estudiante.

- Contratar un profesor particular que le ayude a realizar sus actividades académicas. 


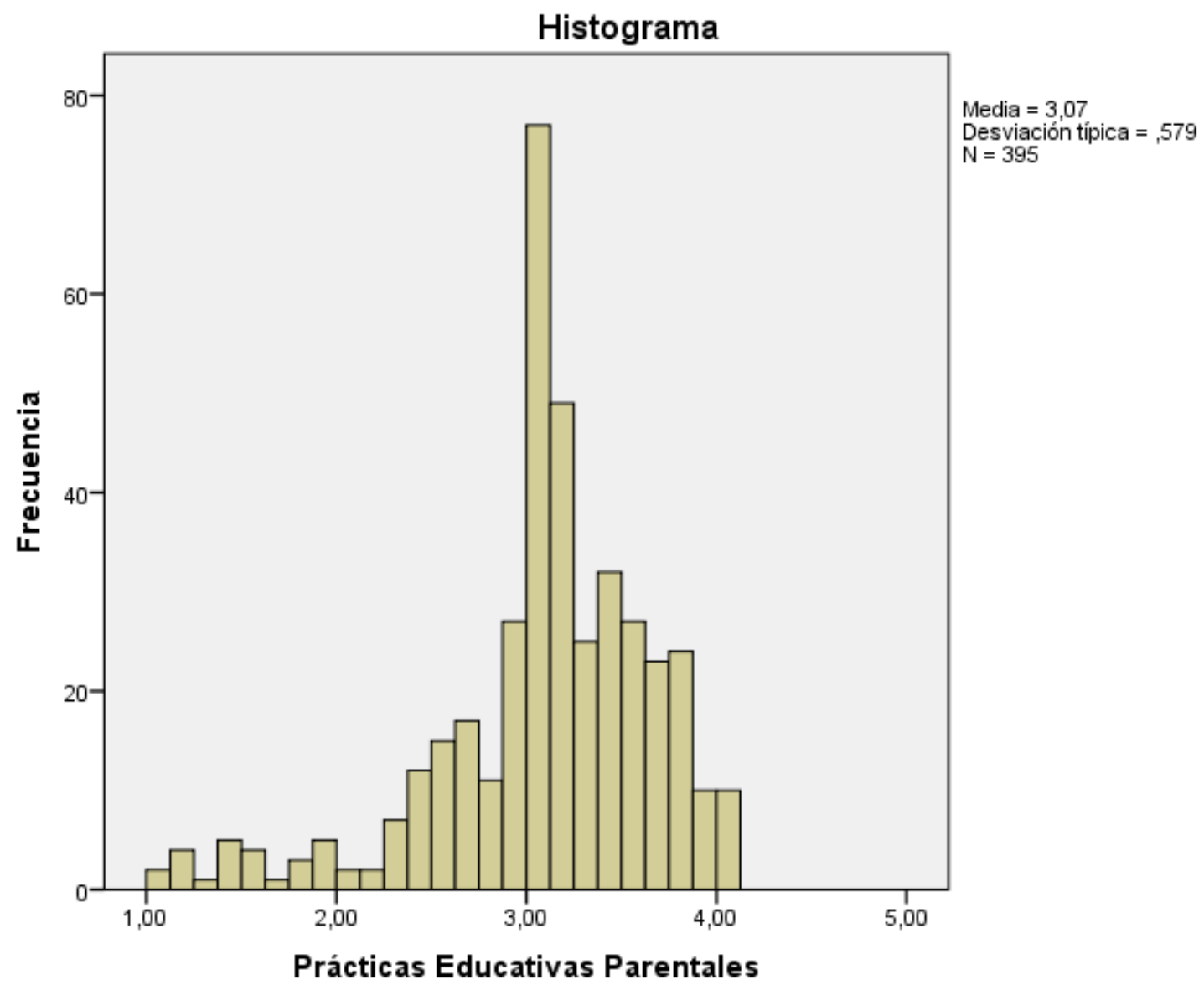

Ilustración 4: Índice de Prácticas Educativas Parentales

El histograma muestra que la concentración de los datos de este índice se encuentra entre el punto 3 y el punto 4. La media alcanza 3,07 y las desviación típica 0,579.

\section{Análisis de Regresión}

Para el análisis de los resultados se utilizó un modelo de regresión lineal general cuya variable dependiente se encuentra en logaritmos y sus variables independientes son ordinales o de escala dependiendo del caso.

Tabla 4: Análisis de Varianza

\begin{tabular}{|c|c|c|c|c|c|c|}
\hline \multicolumn{7}{|c|}{ ANOVA $^{\mathrm{a}}$} \\
\hline \multicolumn{2}{|c|}{ Modelo } & Suma de cuadrados & $\mathrm{Gl}$ & Media cuadrática & $\mathrm{F}$ & Sig. \\
\hline 1 & Regresión & 9,465 & 8 & 1,183 & 40,294 &, $000^{\mathrm{b}}$ \\
\hline & Residual & 11,069 & 377 & ,029 & & \\
\hline & Total & 20,534 & 385 & & & \\
\hline
\end{tabular}


La Tabla 4 muestra el análisis de varianza de la regresión. Como puede observarse, el modelo es significativo lo que implica que las variables independientes utilizadas si están explicando a la variable dependiente. El ajuste del modelo fue de 0,46.

Tabla 5: Regresión

\begin{tabular}{|c|c|c|c|c|c|c|}
\hline \multicolumn{2}{|c|}{ Modelo } & \multicolumn{2}{|c|}{$\begin{array}{l}\text { Coeficientes no } \\
\text { estandarizados }\end{array}$} & \multirow{2}{*}{$\begin{array}{c}\begin{array}{c}\text { Coeficientes } \\
\text { tipificados }\end{array} \\
\text { Beta }\end{array}$} & \multirow{3}{*}{$\begin{array}{r}{ }^{\mathrm{t}} \\
- \\
1,823\end{array}$} & \multirow{3}{*}{$\begin{array}{l}\text { Sig. } \\
\text {,069 }\end{array}$} \\
\hline & & B & Error típ. & & & \\
\hline 1 & (Constante) &,- 181 & ,099 & & & \\
\hline & Edad del encuestado & ,002 & ,001 &, 059 & 1,545 & ,123 \\
\hline & Nivel de Educación & ,065 & ,025 & ,125 & 2,627 & ,009 \\
\hline & Ingreso Promedio Mensual &,- 022 & ,009 &,- 117 & 2,428 &, 016 \\
\hline & $\begin{array}{l}\text { Frecuencia de Lectura del } \\
\text { Apoderado }\end{array}$ &, 025 & ,004 & ,269 & 6,682 &, 000 \\
\hline & Nivel de Integración del Hogar & 041 & 017 &, 105 & 2,418 &, 016 \\
\hline & $\begin{array}{l}\text { Disposición Parental Hacia la } \\
\text { Escuela }\end{array}$ & ,098 & ,016 &, 250 & 6,010 &, 000 \\
\hline & Aspiraciones Parentales &, 114 & ,018 &, 259 & 6,489 &, 000 \\
\hline & $\begin{array}{l}\text { Nivel de Exigencia con los } \\
\text { Hijos }\end{array}$ & ,036 & ,006 &, 255 & 6,243 &, 000 \\
\hline
\end{tabular}

Como puede observarse en la Tabla 5, la edad del encuestado no es significativa, lo cual implica que no existe evidencia suficiente para indicar que la edad de los padres o apoderados es determinante del nivel de prácticas educativas.

El nivel de educación de los padres y la frecuencia de lectura del apoderado se muestran significativas y con signo positivo en el análisis. Específicamente, por cada nivel educativo que alcanzan los padres, las prácticas educativas sobre sus hijos se incrementan en un 6,5\%. Así mismo, por cada incremento unitario en el índice de lectura del apoderado, las PEF crecen en un $2,5 \%$.

El nivel de ingreso es significativo y presenta signo negativo. Esto implicaría que a medida que una persona incrementa su nivel económico, reduce en 2,2\% su nivel de PEF. En contraste, el nivel de integración del hogar se muestra significativo y con coeficiente positivo; esto implica que por cada incremento unitario en el nivel de integración del hogar, o entendiéndose de otra forma como el incremento en las actividades de integración del hogar, se generan incrementos en los niveles de prácticas educativas.

En cuanto a los otros componentes de las EEF, la disposición parental hacia la escuela, las aspiraciones parentales y el nivel de exigencia; los tres índices se muestran significativos y 
positivos, aunque con diferente magnitud. El incremento en el índice de Disposición Parental que se daría por el aumento de actividades relacionadas a este componente, tendría un efecto positivo del 9,8\% sobre las prácticas educativas. Mientras que el incremento en el índice de aspiraciones parentales tiene un efecto positivo del 11,4\% sobre las PEF. Por último, el nivel de exigencias de los padres se relaciona positivamente con las prácticas educativas y tiene un efecto de 3,6\%.

\section{Conclusiones}

Acorde se pudo observar en la sección de resultados, los componentes de capital económico - cultural, la disposición parental y las aspiraciones parentales; explican el nivel de prácticas educativas que utilizan los padres con sus hijos. Entre los puntos más importantes a destacar se pueden mencionar los resultados en la edad, el nivel de ingreso y las aspiraciones.

Según se observó, la edad no es determinante de las PEF, o al menos no existe evidencia estadística suficiente para asegurar que sí lo es. Esto implicaría que existe la posibilidad de que no exista diferencia en el nivel de prácticas educativas de un padre o una madre, independientemente de la edad que tengan. Esta hipótesis debería testearse en una investigación posterior.

En lo que respecta al ingreso de la familia, se mostró significativo y negativo. Por alguna razón, a medida que las familias incrementan su nivel económico, se reducen el nivel de PEF. Esta investigación no indagó sobre las causas de este resultado, siendo línea de partida para futuras investigaciones en las que podrían cuestionarse temas como desinterés o falta de tiempo debido a los trabajos.

Finalmente, se pudo observar que el componente que mayor efecto tiene sobre las PEF son las aspiraciones parentales; lo cual prueba la idea de un trabajo conjunto de escuela y familia; puesto que los padres tienen expectativas altas en las escuelas, pero realizan sus portes desde sus casas.

\section{Bibliografía}

Bernard Van Leer Foundation. (2009). La Primera Infancia en Perspectiva: Programas eficaces para la primera infancia. Obtenido de The Open University: https://bernardvanleer.org/app/uploads/2016/03/Programas_eficaces_para_la_primera_inf ancia2ba1.pdf

Blanco, R. (2012). Una atención y educación de calidad en la primera infancia puede hacer la diferencia. Docencia No. $48,6$.

Bourdieu, P., \& Passeron, J. C. (1977). La Reproducción: Elementos para una teoría de enseñanza. Barcelona: Laia.

Bueno, E. (1994). Fundamentos de Economía y Organización Industrial. Mc Graw Hill.

Campos, A. L. (2010). La Neuroeducación: una nueva aliada para los programas de atención y educación de la primera infancia. En A. L. Campos, Primera Infancia: Una mirada desde 
la Neuroeducación (págs. 11-18). Lima: Cerebrum: Centro Iberoamericano de Neurociencias, Educación y Desarrollo Humano.

CDE. (2010). Departamento de Educación de California. Obtenido de Guias para programas de aprendizaje y desarrollo infantil: http://www.cde.ca.gov/sp/cd/re/documents/itproggdlnesspan.pdf

Contreras, D., Figueroa, J., \& Espina, M. (2012). FAMILIA-ESCUELA Trabajando Juntos. Santiago: Fondo de las Naciones Unidas para la Infancia y Fundación Collahuasi.

DABS. (2006). Folleto Documento Pautas y Prácticas de Crianza. Obtenido de Departamento Administrativo de Bienestar Social del distrito de Bogotá: file://C:/Users/sybaq_000/Downloads/Folleto-Pautas-practicas-crianza2\%20(1).pdf

Early Childhood Learning \& Knowledge Center. (15 de Agosto de 2011). Marco de participación en Head Start de los padres, la familia y la comunidad (PFCE). Obtenido de Se promueve la participación familiar y la preparación para la escuela desde la etapa prenatal hasta la edad de 8 años: http://eclkc.ohs.acf.hhs.gov/hslc/Espanol/participacion/docs/pfceframework-spanish.pdf

Epstein, J. (2013). Programas efectivos de Involucramiento familiar en las escuelas: estudios y prácticas. Santiago: Fundación CAP.

García, F. (29 y 30 de Noviembre de 2001). Modelo Ecológico / Modelo Integral de Intervención en atención Temprana. Madrid, España.

Gubbins, V. (2014). Estrategias educativas de familias de clase alta: un estudio exploratorio. Psyche. Versión online , 1-17.

Gubbins, V. (2012). Familia y escuela: tensiones, reflexiones y propuestas. Docencia No. 46 , 6473.

Gubbins, V., \& Ibarra, S. (Mayo de 2016). Estrategias Educativas Familiares en Enseñanza Básica: Análisis Psicométrico de una Escala de Prácticas Parentales. Psykhe vol.25 no.1 , 1-17.

Hoel, P., \& Jessen, R. (1983). EStadística Básica para Negocios y Economía. CECSA.

López, M. E., \& Caspe, M. (Junio de 2014). Involucramiento Familiar para el aprendizaje en cualquier momento y lugar. Obtenido de Harvard Family Research Project: http://www.hfrp.org/

Ministerio de Educación, C. y. (16-18 de Abril de 2015). Las relaciones entre familia y escuela. Experiencias y buenas prácticas. Documento base. Obtenido de XXIII Encuentro de Consejos Escolares Autonómicos y del Estado: http://www.mecd.gob.es/dctm/cee/encuentros/23encuentro/23encuentroceaedocumentoba se2015.pdf?documentId=0901e72b81 cba426 
Rivera, M., \& Milicic, N. (2006). Alianza Familia-Escuela: Percepciones, Creencias, Expectativas y Aspiraciones de Padres y Profesores de Enseñanza General Básica. Psykhe , 1-14.

Sallés, C., \& Ger, S. (2011). Las competencias parentales en la familia contemporánea: descripción, promoción y evaluación. Educación Social: Revista de Intervención Socioeducativa , 25-47.

Solís, D. (2013). El Buen Vivir empieza en la Infancia. MIESpacio - Revista del Ministerio de Inclusión Económica y Social , 3.

UNICEF. (Septiembre de 2014). La niñez y la adolescencia en el Ecuador contemporáneo: avances y brechas en el ejercicio de derechos. Obtenido de Observatorio Social del Ecuador:

http://www.unicef.org/ecuador/NA_EcuadorContemporaneo_ultima_version.pdf

Weiss, H., Bouffard, S., Bridgall, B., \& Edmund, G. (Diciembre de 2009). Hacia un nuevo enfoque sobre el involucramiento de la familia en la educación: apoyar a las familias para promover la equidad en la educación. Obtenido de Harvard Family Research: http://www.hfrp.org/publications-resources/browse-our-publications/reframing-familyinvolvement-in-education-supporting-families-to-support-educational-equity 